\title{
The Theory of Relativity is an Error in Physics; the Speed of Light excludes the Laws of Optics
}

\author{
Jozef Babiak
}

ABSTRACT

Physicists measured the propagation of light in vacuum and matter through experiments with light over the course of several centuries. The Laws of Optics were derived from these measured results. The Laws of Optics were verified repeatedly via real experiments with light and used in the calculations of optical instruments that work properly. The Laws of Optics accurately define the propagation of light in vacuum and matter. All the theories of the propagation of light that ignore the Laws of Optics can, therefore, be considered an error in physics.

The theories and laws of propagation of light in matter were written by famous physicists, such as Isaac Newton (1670), Christiaan Huygens (1650), Augustin-Jean Fresnel (1819), and Thomas Young (1800). The law of propagation of light in matter was described by Snell in 1620. Snell's law is the law of the light refractive index in matter. Optical instruments are calculated according to the Laws of Optics, so by their correct operation they confirm the validity of the Laws of Optics.

Two hundred years ago, André-Marie Ampére expressed the basic approach in discovering the laws of physics: "First observe the facts, to vary their circumstances as much as possible, to accompany this first task with exact measurement so as to deduce from them the general laws based solely upon experience, and to deduce from these laws, independently of any hypothesis on the nature of the forces that produce the phenomena, the mathematical value of these forces". This approach to the measurement of the light propagation is now used in all the laboratories around the world and, therefore, can be considered as objective and accurate.

Keywords: Speed of Light, Excludes the Laws of Optics

\section{*Correspondence to Author:} Jozef Babiak

How to cite this article:

Jozef Babiak. The Theory of Relativity is an Error in Physics; the Speed of Light excludes the Laws of Optics. American Journal of Scientific Research and Essays, 2018:3:2.

\section{eScîPub}

eSciPub LLC, Houston, TX USA. Website: http://escipub.com/ 


\section{Theory of Relativity and the Laws of Optics}

Isaacson in his work [1] describes the life and scientific career of Einstein in detail.The contribution of the book is the realistic portrayal of the events from Einstein's life derived from the original documents. On page 21 of the work [1], he mentions: "As with the special theory of relativity, also in this case his thinking had evolved through thought experiments." On page 23, he continues: "Einstein discerned great principles through thought experiments rather than by methodical inductions based on the experimental data." In physics, an argument can be considered as valid only if the measured values are gained in a fairly performed experiment. The thought experiment is just the subjective opinion of one person on the physical experiment; its veracity must be verified through real measurement in the experiment. Isaacson states: "Einstein never did any measurements with an interferometer", which explains why he didn't apply the Laws of Optics in the calculation of the propagation of light in matter and in the measurements with the interferometers. Einstein in the description of the light propagation in the Michelson-Morley experiment did not consider the Law of the light refractive index in air and this was Einstein's error in his Theory of Relativity.

Einstein in his book; The Theory of Relativity [2], on page 96, in the section dealing with light propagation in air refers to the speed of light in air along its trajectory: "Of course we must refer to the process of the propagation of light (and indeed every other process) as a rigid reference-body (co-ordinate system). As such a system lets us choose our embankment. We shall imagine the air above it to have been removed. "A ray of light will be sent along the embankment..." He continues: "If every ray of light is propagated relative to the embankment with the velocity $c$..." This description of the light propagation on the embankment is false. Einstein's assumption "We shall imagine the air above it to have been removed..." is only Einstein's thought. It is not possible to create a vacuum around the embankment; vacuum can only be produced in a laboratory. In the described calculation of the time in which the light passes along the trajectory in the air the law of the light refractive index must be taken into account, where the speed of light is $\mathbf{c} \mathbf{n}$, not c. This is Einstein's error in his Theory of Relativity!

My measurement of the speed of light with the Michelson interferometer takes into account the Law of the Optics. The results are published in an article available at: http://www.babiak.websnadno.cz/Meraniezmeny-abs-indexu-lomu-vzduchu-.html.

In the measurement of the speed of light with the Michelson interferometer it is easy to ascertain that in the interferometer arms is air and, therefore, in the interferometer arms the speed of light is "c/n", not "c" as calculated in the Theory of Relativity. The presence of the air in the interferometer arms is confirmed by the shift of the interference fringes while causing the airflow in the interferometer arm. Throughout the entire time of the slow rotation of the interferometer in the Michelson-Morley experiment, the speed of light was identical and constant in both interferometer arms and equal to "c/ $\mathbf{n}$ ". Therefore, the shift of the interference fringes was not observed. It was not caused by the shortening of the interferometer arm as calculated in the Theory of Relativity. Einstein's idea about the direction of the interferometer arms' movement in ether is not correct, as he did not recognise the existence of ether.

Einstein in his work [3] on page 110, quotes a speech in London: "The law of a constant speed of light in vacuum with the same value in all inertial frames of reference, as documented by developments in electrodynamics and optics and proven by the famous Michelson experiment (principle of special relativity), has resulted in the fact that the concept of time must be regarded as.....". In optics, the speed of light in matter is calculated respecting the Law of the light refractive index. The results measured in the Michelson-Morley experiment 
cannot prove a constant value of speed of light in vacuum since the experiment was performed in the air. In the experiment, there was no vacuum and that's why the calculation with the speed of light in vacuum in the MichelsonMorley experiment is Einstein's error!

I have measured the speed of light in vacuum with the Michelson interferometer by taking into account the Laws of Optics. The measurement is described in an article published in the American Journal of Scientific Research (ASRE, 2016, Vol. 1) available at: http://escipub.com/Articles/AJSRE/Vol1/BabiakAJSRE-2016.

In the paper, I describe the measurement of the speed of light with an interferometer in a vacuum, in which I compare the speed of light of a helium laser in a vacuum with the speed of light of a green laser with a different light wavelength in a vacuum. The measurement unequivocally shows that the speed of light in a vacuum is different with a different light wavelength in a vacuum.

Einstein in his calculation of shift of the interference fringes in the Michelson-Morley experiment states that the speed of light in an interferometer arm is calculated as $\mathbf{c + v}$, which is in contradiction with the law of the constant speed of light on which Einstein made the basis for his Theory of Relativity. The MichelsonMorley experiment was performed in air, and the speed of light in the interferometer arms is according to the Law of light refraction index in air, the same on both arms of the interferometer arms and referred to as $\mathbf{c} / \mathbf{n}$ and not calculated as $\mathbf{c}$. Throughout the entire time of the rotation of the interferometer, the speed of light was identical and constant in both inter-ferometer arms and equal to $\mathbf{c} / \mathbf{n}$. Therefore, the phase shift of rays of light from the interferometer arms and the resulting shift of interference fringes were not observed! If we calculate the speed of light according to the Laws of Optics, no contraction of the length of the interferometer's arms can be observed from the calculation of the propagation of light. In order to explain why the interference fringes were not shifted during the interferometer rotation, Lorentz stated the contraction of the arm of the interferometer in the direction of interferometer movement relative to ether. Einstein in his work [2] on page 119 cites: "Guided by purely formal points of view, H. A. Lorentz was the first to introduce that the particles constituting the electron experience a contraction in the direction of motion..." In the Theory of propagation of light, Lorentz ignores the Laws of Optics!

Einstein in his book, The Theory of relativity [2] on page 121 states: "According to this there is no outstanding co-ordinate system, which gives rise to the introduction of the idea of ether and so there is no ether wind and no experiment, which would lead to its evidence". Einstein in his calculation of shift of the interference fringes in the Michelson-Morley experiment counts with ether but at the same time he denies the existence of ether. Therefore, Einstein is contradicting himself! In optics, ether was never measured in a real experiment with light; in the calculation of optical instruments it is not used because the Laws of Optics do not recognise the ether and do not count with ether!

The reference to the Theory of Relativity based on the results measured during the measurement of the light refractive index with interferometers is described in the article available on the website [7]. AC converters were used to scan the shifts of the interference fringes on ground glass by using photo transistors and they were entered into computer memory in order to avoid mistakes in the calculation of the shifted interference fringes. With the help of a number of shifted interference fringes on interferometer ground glass, the index of light refractive index and the wavelength of light in matter is calculated. I converted, by the double interferometers on the principle of the comparison of the speed of light in vacuum with the speed of light in matter, all of the measurements of light refractive index and light wavelength in vacuum and matter. 


\section{Summary}

Einstein wrote his Theory of Relativity as a doctor (PhD) of sciences, so he must have known the Laws of Optics. Failure to apply the Laws of Optics in the Theory of Relativity is a major error in physics.

1. Einstein did not perform any measurements of the speed of light by interferometers. He discerned the propagation of light through thought experiments rather than by Laws of Optics.

2. In his thought experiments with light, he did not count on the existence of air and on the absolute light refractive index in the air according to the Laws of Optics.

3. He pronounced the axiom of the constant speed of light in a vacuum without measurement; he counted with the speed of light as $\mathbf{c}+\mathbf{v}$, where $\mathbf{v}$ is the speed of light in ether, yet he did not recognise the existence of ether.

My view on the Theory of Relativity is based on more than 20 years of measurements with interferometers and 10 years of studies of works regarding interferometry that are available on the Internet. All of the values were measured with interferometers and in all the calculations the Laws of Optics were taken into account. Many years of practical experience in the measurement of the speed of light with interferometers gave me the opportunity to understand the validity of the Law of light refractive index in light propagation in interferometers.

\section{References:}

1. Walter Isaacson: Einstein jeho život a vesmír, PASEKA, Praha-Litomyšl, 2010

2. Albert Einstein: Teórie relativity, VUTIUM, Brno 2005

3. Albert Einstein: Jak vidím svět, Lidové noviny, Praha, 1993

4. Edlén, B.: The Refractive Index of Air. Metrologia, Vol. 2, 1966

5. http://www.babiak.websnadno.cz/Novy-principmerania-rychlosti-svetla-dv.html

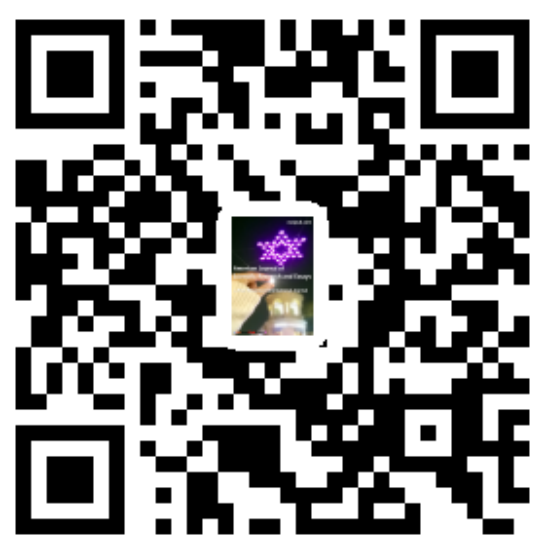

